\title{
Barium Strontium Bismuth Niobate Layered Perovskites: Dielectric, Impedance and Electrical Modulus Characteristics
}

\author{
M.P. Dasari ${ }^{a}{ }^{*}$, K. Sambasiva RaO ${ }^{b}$, P. Murali Krishna ${ }^{b}$ And G. Gopala Krishna ${ }^{a}$ \\ ${ }^{a}$ Department of Physics, Gitam Institute of Technology, Gitam University \\ Visakhapatnam-530 045, India \\ ${ }^{b}$ Centre for Piezoelectric Transducer Materials, Physics Department, Andhra University \\ Visakhapatnam-530 003, India
}

(Received September 19, 2010; in final form November 30, 2010)

\begin{abstract}
$\mathrm{SrBi}_{2} \mathrm{Nb}_{2} \mathrm{O}_{9}(\mathrm{SBN})$ is a bismuth layered perovskite compound, due to its relatively high Curie temperature, has potential application as high ceramic transducer. Also, it is an attractive ferroelectric material that is being considered in non-volatile random access memory cells. Present article describes preparation, dielectric, impedance and modulus characteristics. Temperature and frequency dependence of dielectric permittivity, impedance and electric modulus of barium strontium bismuth niobate $\left(\mathrm{Ba}_{0.1} \mathrm{Sr}_{0.9} \mathrm{Bi}_{2} \mathrm{Nb}_{2} \mathrm{O}_{9}, \mathrm{BSBN}\right)$ have been studied in the range of $35-590^{\circ} \mathrm{C}$ and $45 \mathrm{~Hz}-5 \mathrm{MHz}$, respectively. The structural analysis of compound revealed orthorhombic at room temperature. Complex impedance Cole-Cole plots are used to interpret the relaxation mechanism. These plots shows the relaxation behavior as non-Debye type. By using the Cole-Cole plots grain and grain boundary contributions towards conductivity have been estimated. From electrical modulus formalism polarization and conductivity relaxation behavior in BSBN have been discussed. DC and AC conductivity measurements have been performed on BSBN.

PACS: 77.84.--s, 77.84.Ek, 72.80.Ng
\end{abstract}

\section{Introduction}

Oxides of the Aurivillius [1] family constitute an important class of materials for their ferroelectric properties. They are of substantial interest for integrated circuit memories [2], high temperature piezoelectric sensors [3], electrooptic modulators and displays [4].

Currently, isotropic perovskite ferroelectrics $\mathrm{Pb}(\mathrm{Zr}, \mathrm{Ti}) \mathrm{O}_{3} \quad(\mathrm{PZT})$ and Bi-layer structured ferroelectrics (BLSFs), $\mathrm{SrBi}_{2} \mathrm{Nb}_{2} \mathrm{O}_{9}$ (SBN), $\mathrm{BiTi}_{3} \mathrm{O}_{12}$ (BIT) and $\mathrm{SrBi}_{2} \mathrm{Ta}_{2} \mathrm{O}_{9}$ (SBT), are known as useful ferroelectric materials [5-12]. For practical ferroelectric random access memory (FRAM) application, it is necessary to develop a new ferroelectric material with a high remanent polarization, high fatigue endurance, low leakage current polarization, low leakage current and low processing temperature $[5-8,13,14]$.

Bismuth oxide layered perovskites (BOLP) are generally formulated [15] as $\left(\mathrm{Bi}_{2} \mathrm{O}_{2}\right)^{2+}\left(\mathrm{A}_{m-1} \mathrm{~B}_{m} \mathrm{O}_{3 m+1}\right)^{2-}$. They consist of an intergrowth between $\left(\mathrm{Bi}_{2} \mathrm{O}_{2}\right)^{2+}$ sheets and $\left(\mathrm{A}_{m-1} \mathrm{~B}_{m} \mathrm{O}_{3 m+1}\right)^{2-}$ perovskite-like layers, with $m$ being the number of octahedra stacked along the direction perpendicular to the sheets, and $\mathrm{A}$ and $\mathrm{B}$ are the 12-fold and six-fold coordination sites of the perovskite slab, respectively. More often $\mathrm{A}$ is $\mathrm{Bi}^{3+}, \mathrm{Ba}^{2+}, \mathrm{Sr}^{2+}$, $\mathrm{Ca}^{2+}, \mathrm{Pb}^{2+}, \mathrm{Bi}^{3+}, \mathrm{K}^{+}$or $\mathrm{Na}^{+}$; $\mathrm{B}$ is $\mathrm{Ti}^{4+}, \mathrm{Nb}^{5+}, \mathrm{Ta}^{5+}$, $\mathrm{Mo}^{6+}, \mathrm{W}^{6+}$ or $\mathrm{Fe}^{3+}[15]$.

The ac impedance analysis is known to be a powerful means to separate out the grain boundary and grain-electrode effects, which usually are the sites of trap for

* corresponding author; e-mail: rajadasari78@rediffmail.com oxygen vacancies and other defects. It is also useful in establishing space charge polarization and its relaxational mechanism, by appropriately assigning different values of resistance and capacitance to the grain and grain boundary effects. A noteworthy aspect of the impedance analysis is the possibility of calculating the different contributions to the conductivity, namely the bulk, grain boundary and grain-electrode contributions.

In view of the importance of ac impedance analysis, the present paper describes a detailed analysis of dielectric and impedance spectroscopy studies which have been carried out in barium substituted strontium bismuth niobate (BSBN). No impedance analysis on BSBN has been found in literature.

\section{Experimental}

Barium substituted $\mathrm{SrBi}_{2} \mathrm{Nb}_{2} \mathrm{O}_{9}$ ceramic specimens have been prepared by the multi-step mixed oxide route. $\mathrm{Bi}_{2} \mathrm{O}_{3}, \mathrm{SrCo}_{3}, \mathrm{BaCo}_{3}$ and $\mathrm{Nb}_{2} \mathrm{O}_{5}$ are used as starting materials. These are thoroughly mixed for $6 \mathrm{~h}$ and calcined at $900{ }^{\circ} \mathrm{C}$ for $2 \mathrm{~h}$. The calcined powder has been crushed thoroughly and pelletized into a disk using polyvinyl alcohol as binder. Finally, the disks have been sintered at $1150{ }^{\circ} \mathrm{C}$ for $1 \mathrm{~h}$.

The phase formation was confirmed by X-ray diffraction (XRD) studies. The X-ray diffractogram of the sample has been taken using $\mathrm{Cu} K_{\alpha}$ radiation. For the dielectric characterization, the sintered disk (of thickness of $2 \mathrm{~mm}$ and diameter of $12 \mathrm{~mm}$ ) has been polished, then coated with a conducting silver paste for making electrical contacts. The disk was dried at $600{ }^{\circ} \mathrm{C}$ for 30 min. High temperature dielectric measurements and impedance measurements have been carried out using an 
LCR meter (computer interfaced HIOKI 3532-50 LCR Hitester) from $42 \mathrm{~Hz}$ to $5 \mathrm{MHz}$ in the temperature range from 35 to $590^{\circ} \mathrm{C}$.

\section{Results and discussion}

\section{1. $X R D$}

Figure 1 shows the X-ray diffraction pattern of the sample taken at room temperature. All the peaks of the
X-ray profile are indexed, and lattice parameters have been determined by using the standard computer program (POWD). A good agreement between the observed and calculated interplanar spacing ( $d$ values) suggests the compound has orthorhombic structure at room temperature with lattice parameters $a=5.518 \AA, b / a=1.000$ and $c=25.12 \AA$. X-ray diffraction confirms that the specimen is single phase.

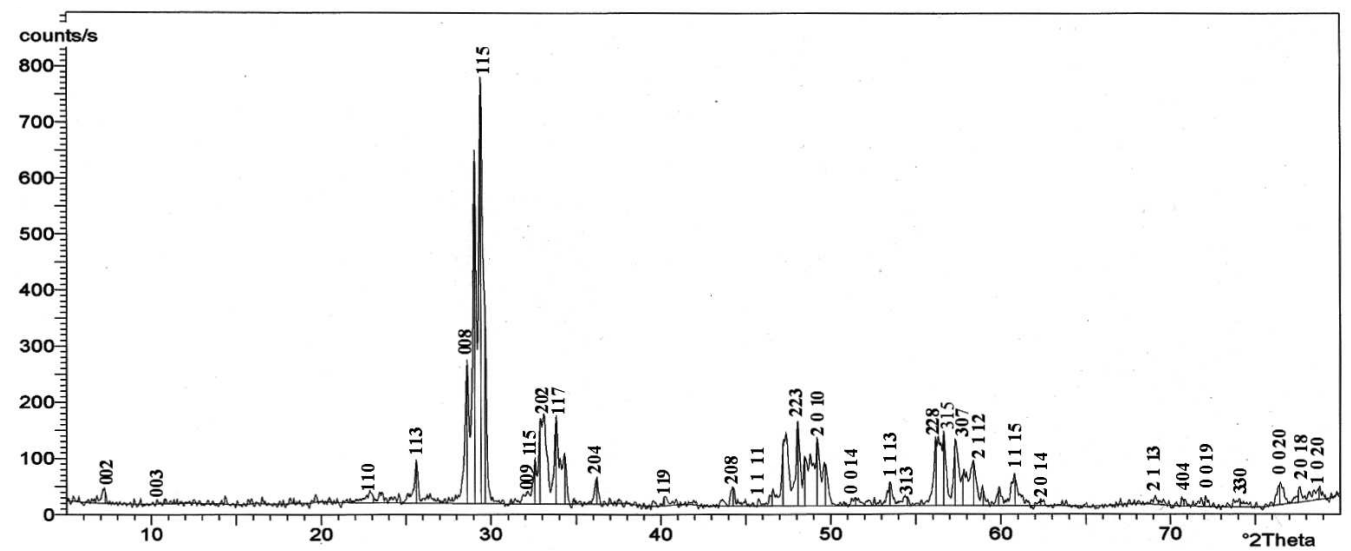

Fig. 1. X-ray diffractogram of BSBN.

\subsection{Dielectric}

Figure $2 \mathrm{a}$ shows the variation of the real $\left(\varepsilon^{\prime}\right)$ part of dielectric constant with temperature at various frequencies for BSBN. The dielectric constant with temperature on BSBN shows a broadened maximum around $460{ }^{\circ} \mathrm{C}$, indicating the ferroelectric phase transition $\left(T_{\mathrm{c}}\right)$. Inset of Fig. 2a shows the temperature dependence of $\varepsilon^{\prime}$ of SBN ceramic. The $T_{\mathrm{c}}$ of SBN ceramic has been found to be $392{ }^{\circ} \mathrm{C}$. Smolenskii et al. [16] discovered a phase transition taking place at $420^{\circ} \mathrm{C}$ by the measurement of dielectric constant, while Subba Rao [17] found the phase transition taking place at $440^{\circ} \mathrm{C}$ in SBN. Figure $2 \mathrm{~b}$ shows the variation of imaginary part $\left(\varepsilon^{\prime \prime}\right)$ of dielectric constant with temperature at various frequencies. The $\varepsilon^{\prime \prime}$ does not show clear anomaly for the sample, whereas only mere slope changes near $T_{\mathrm{c}}$ occur for all frequencies.

Figure 3 shows the variation $\varepsilon^{\prime}$ and $\varepsilon^{\prime \prime}$ with frequency at different temperatures. From Fig. 3a both the values of $\varepsilon^{\prime}$ and $\varepsilon^{\prime \prime}$ almost start at approximately 50 at $45 \mathrm{~Hz}$ and $280^{\circ} \mathrm{C}$. As the temperature increases to $360^{\circ} \mathrm{C}, \varepsilon^{\prime}$ increases to 100 , whereas $\varepsilon^{\prime \prime}$ increases to above 200 , and they intersect at a frequency $1 \mathrm{kHz}$. With further increase in temperature both $\varepsilon^{\prime}$ and $\varepsilon^{\prime \prime}$ values are increased. As the temperature increases above $440{ }^{\circ} \mathrm{C}, \varepsilon^{\prime}$ and $\varepsilon^{\prime \prime}$ intersect at a particular frequency. The intersection frequency shifts towards the higher frequency side as temperature increases. The sharp rise of $\varepsilon^{\prime}$ and $\varepsilon^{\prime \prime}$ at low frequencies is most probably due to conducting ion motion. When dealing with a charge carrier system in high

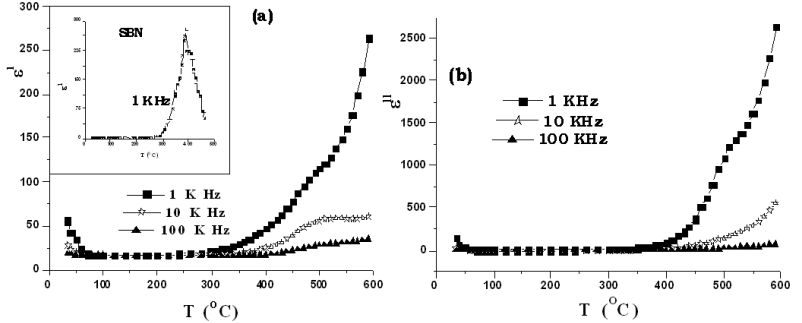

Fig. 2. Variation of the (a) real $\left(\varepsilon^{\prime}\right)$, and (b) imaginary $\left(\varepsilon^{\prime \prime}\right)$ parts of dielectric constant of BSBN with temperature.

conducting material, it is preferable to plot the ac complex impedance or conductivity instead of the dielectric loss for characterizing ferroelectric materials.

Further, both the $\varepsilon^{\prime}$ and $\varepsilon^{\prime \prime}$ exhibit high value which reflects the effect of space charge polarization and/or conducting ion motion. In the conducting dielectric materials, high $\varepsilon^{\prime}$ values may be related to the accumulation of charges at the interface between the sample and electrodes, i.e. space charge polarization. The value of $\varepsilon^{\prime \prime}$ at low frequency becomes very high due to free charge motion within the material and are connected to ac conductivity relaxation. A further analysis of the dielectric behavior would be more successfully achieved by using the formulation of dielectric modulus spectroscopy which suppresses the effect of electrode polarization or mobile ion polarization. 

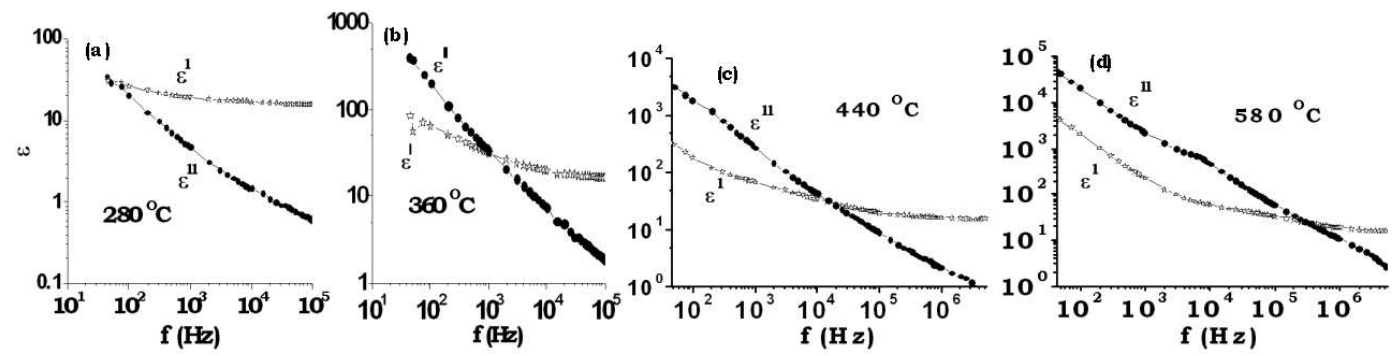

Fig. 3. Variation of real and imaginary dielectric constant as a function of frequency at several temperatures.

\subsection{Frequency dependence of the dielectric constants $\varepsilon^{\prime}$ and $\varepsilon^{\prime \prime}$}

The frequency dependence of the real $\left(\varepsilon^{\prime}\right)$ and imaginary $\left(\varepsilon^{\prime \prime}\right)$ parts of the dielectric constant on a log-log scale at different temperatures are shown in Fig. 3. Both $\varepsilon^{\prime}$ and $\varepsilon^{\prime \prime}$ show strong dispersions at low frequencies. This event is attributed to the low frequency space charge accumulation effect. Such strong dispersions in both the components of the complex dielectric constant appear to be a common feature in ferroelectrics associated with non-negligible ionic conductivity and is referred to as the low frequency dielectric dispersion (LFDD) [18, 19]. Detailed studies of this phenomenon were carried out by Jonscher et al. [20].

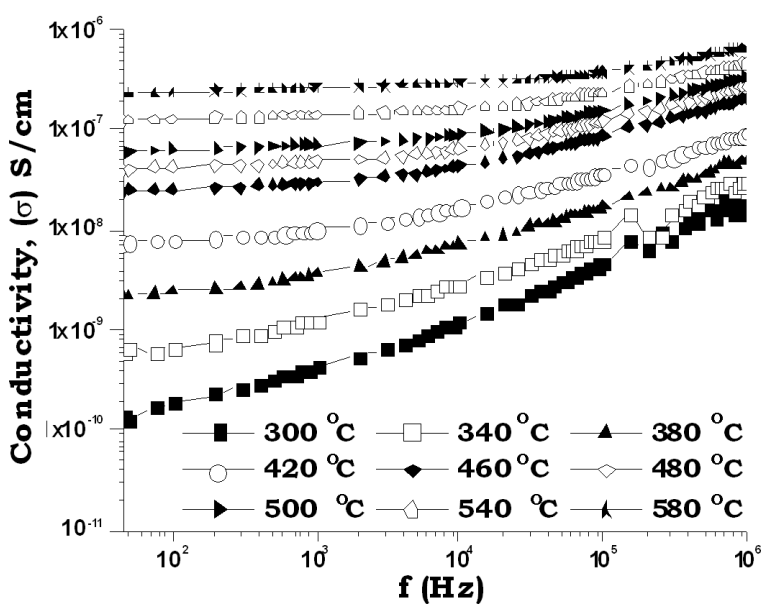

Fig. 4. Double logarithmic plot of ac conductivity vs. frequency at several temperatures.

The dispersion in $\varepsilon^{\prime \prime}$ is stronger than that in $\varepsilon^{\prime}$ implying that it is influenced by dc conductivity. The low frequency slope of the curve $\log \varepsilon^{\prime \prime}$ vs. $\log f$ is close to -1 indicating the predominance of the dc conduction in this frequency region. Figure 4 (discussed in dc and ac conductivity) shows the variation of the real part of the ac conductivity as a function of frequency at a few elevated temperatures. It indicates the existence of low frequency independent plateau-like region $\left[\sigma_{\mathrm{dc}}\right]$ and subsequently the conductivity increases with increase in fre- quency, varying approximately as a power of frequency $\left(\omega^{n}\right)$ (where $n$ is a function of temperature as well as frequency) at all the temperatures. However, the trend of the curve does not change with temperature; only the onset of the dispersion is shifted to higher frequencies as the temperature is increased. This observation is in line with that reported in the literature for $0.3 \mathrm{Na}_{2} \mathrm{O} 0.7 \mathrm{~B}_{2} \mathrm{O}_{3}$ glass samples by Roling et al. [21].

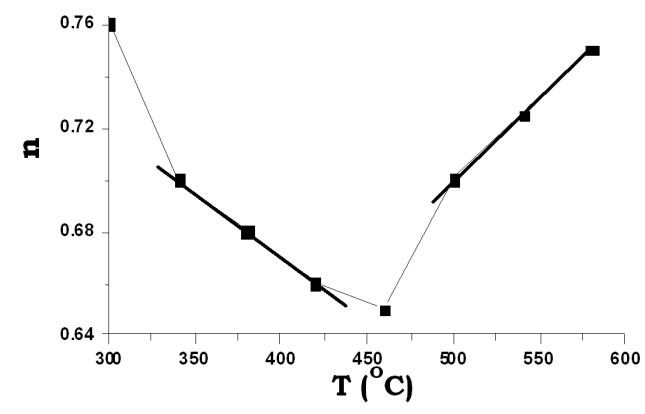

Fig. 5. Variation of critical exponent $n(T)$ with temperature showing a minimum at $T_{\mathrm{c}}$.

Figure 5 shows the temperature dependence of the exponent $n(T)$. An interesting feature of Fig. 5 is the two linear regions corresponding to the paraelectric and ferroelectric states. A linear relation of the exponent with temperature has also been found in some ion conducting systems [22-24].

The interaction between the charge carriers participating in the polarization process is characterized by the parameter $n$. A unit value of $n$ implies a Debye case, and it is attainable [25] at very low temperatures. However, as the temperature increases, the interaction increases, leading to a decrease in $n$. From Fig. 5 the value of $n$ calculated from the high frequency region decreases as the temperature increases and attains a minimum near $T_{\mathrm{c}}$ and subsequently it increases with further increase in temperature. The observed minimum at $T_{\mathrm{c}}$ in BSBN implies the strong interaction between the charge carriers and the lattice.

The complex dielectric constant (Cole-Cole) plots at two different temperatures (above and below $T_{\mathrm{c}}$ ) are shown in Fig. 6. In both the plots the universal ma- 


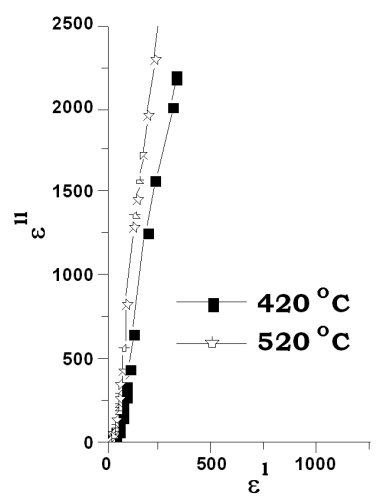

Fig. 6. Cole-Cole plots of complex dielectric constant at 420 and $520^{\circ} \mathrm{C}$.

terial behavior, represented by $\varepsilon_{\infty}+A(T)\left(\omega^{n(T)-1}\right)$ is obeyed by making a straight line inclined at an angle $[1-n(T)] \pi / 2$ and an intercept of $\varepsilon_{\infty}$ with the horizontal axis $[26]$.

\subsection{Dielectric dispersion relations and interpretation of the experimental results}

The complex dielectric constant as a function of the frequency $\omega$ in accordance with the Jonscher power law is given by the following expression:

$$
\begin{aligned}
\varepsilon^{*} & =\varepsilon^{\prime}-\mathrm{i} \varepsilon^{\prime \prime}=\varepsilon_{\infty}+\sigma / \mathrm{i} \varepsilon_{0} \omega \\
& +\left[a(T) / \varepsilon_{0}\right]\left[\mathrm{i} \omega^{n(T)-1}\right],
\end{aligned}
$$

where $\varepsilon_{\infty}$ is the "high frequency" value of the dielectric constant, $n(T)$ is the temperature-dependent exponent and $a(T)$ determines the strength of the polarizability arising from the universal mechanism in question. The real and imaginary parts of the complex dielectric constant are given by the following relations:

$$
\begin{aligned}
& \varepsilon^{\prime}=\varepsilon_{\infty}+\sin (n(T) \pi / 2) \omega^{n(T)-1}\left[a(T) / \varepsilon_{0}\right], \\
& \varepsilon^{\prime \prime}=\sigma / \mathrm{i} \varepsilon_{0} \omega+\cos (n(T) \pi / 2) \omega^{n(T)-1}\left[a(T) / \varepsilon_{0}\right],
\end{aligned}
$$

where the first term in Eq. (2) determines the lattice response and that in Eq. (3) corresponds to the dc conduction part, while the second term in both the equations reflects the charge carrier contribution to the dielectric constant. The temperature and frequency dependences of the dielectric constant $\varepsilon^{\prime}$ (Figs. 2 and 3) could be explained by Eq. (2). The charge carrier term $\left(\sin (n(T) \pi / 2) \omega^{n(T)-1}\left[a(T) / \varepsilon_{0}\right]\right)$ dominates at low frequency and $\varepsilon_{\infty}$ is negligible. Therefore, for a constant $n$, Eq. (2) yields a straight line with a slope equal to $n-1$ in the double logarithmic plot of $\varepsilon^{\prime}$ and frequency. At high frequencies the charge carriers fail to respond to the external field, therefore the measured dielectric constant is due to the contribution from the lattice polarization. This accounts for a linear decrease in the low frequency region and a frequency-independent plateau region at high frequencies (Fig. 3). As $A(T)(a(T))$ increases with increase in temperature [27], the charge carrier term becomes more and more prominent at high temperatures, thereby resulting in the low frequency dielectric dispersion. An anomalously strong dispersion of $\varepsilon^{\prime}$ near $T_{\mathrm{c}}$ suggests the coupling between the charge carriers and the lattice [26]. The behavior of $\varepsilon^{\prime \prime}$ could be explained by Eq. (3).

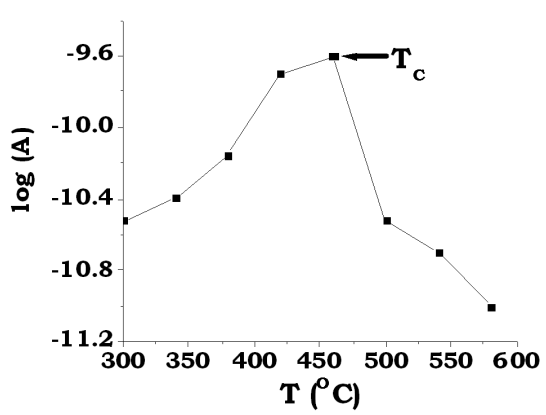

Fig. 7. The temperature dependence of the prefactor $A(T)$ showing a peak at $T_{\mathrm{c}}$.

Careful analysis of Fig. 3 indicates the existence of two slopes corresponding to -1 in the low frequency region and $-(1-n)$ in the high frequency region. As the dc conductivity term increases with increase in temperature, the second term in Eq. (3) is totally over-shadowed by the first term. So at low frequencies and high temperatures, the dc conductivity term dominates and yields a slope of -1 , which indeed is consistent with the data shown in Fig. 3. Figure 7 shows the temperature dependence of the prefactor $A(T)$ showing a peak at $T_{\mathrm{c}}$. The prefactor $A(T)$ which determines the strength of polarizability exhibits a peak at $T_{\mathrm{c}}[18]$.

\subsection{Impedance spectroscopy}

Generally, the impedance properties arise due to intragrain, intergrain and electrode processes. The motion of charges could occur in a variety of ways, namely charge displacement (long-range or short-range), dipole re-orientation, space charge formation etc. The complex impedance $Z^{*}(\omega)$ of the present material can be described by the following equation:

$$
z^{*}=Z^{\prime}(\omega)+\mathrm{j} Z^{\prime \prime}(\omega),
$$

where $Z^{\prime}$ and $Z^{\prime \prime}$ are ascribed to real and imaginary parts of impedance.

Figure $8 \mathrm{a}$ presents the variation of the real part impedance $\left(Z^{\prime}\right)$ vs. frequency using a double logarithmic scale for several temperatures. From Fig. 7a, the $Z^{\prime}$ decreases slowly in the frequency range of $10^{3}-10^{6} \mathrm{~Hz}$, depending on the temperature, and continuously with an increase in frequency.

Figure $8 \mathrm{~b}$ shows the variation of the imaginary part of the impedance $\left(Z^{\prime \prime}\right)$ as a function of frequency at several temperatures. It can be seen that the curves display broad and low intensity peaks. The peak frequency shifts to higher frequency side with increasing temperature, and 


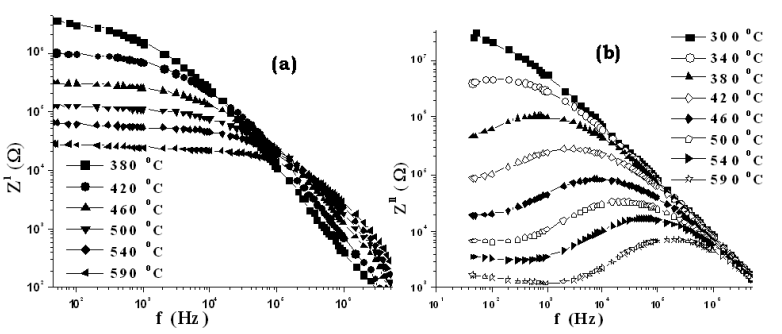

Fig. 8. Frequency dependence of (a) real $\left(Z^{\prime}\right)$, (b) imaginary $\left(Z^{\prime \prime}\right)$ parts impedance.

the relaxation occurs over several decades of frequency. Intuitively one might conclude that all these curves merge at frequencies $\geq 1 \mathrm{MHz}$. The activation energies associated with each peak were determined from the frequencies at which the maxima $\left(f_{\mathrm{p}}\right)$ occur, and it is known that at maxima the equation $\tau=1 / 2 \pi f$ holds well. The activation energy has been determined by using the equation $\tau=B \exp \left(-E_{\mathrm{a}} / k T\right)$, where $B$ is a pre-exponential factor, and $k$ and $T$ have their usual significance. The behavior of the relaxational frequencies follows the Arrhenius law where the activation energies in the temperature regions $340-380^{\circ} \mathrm{C}, 400-460{ }^{\circ} \mathrm{C}$ and $480-580^{\circ} \mathrm{C}$ are $0.61 \mathrm{eV}, 0.59 \mathrm{eV}$ and $0.45 \mathrm{eV}$, respectively.

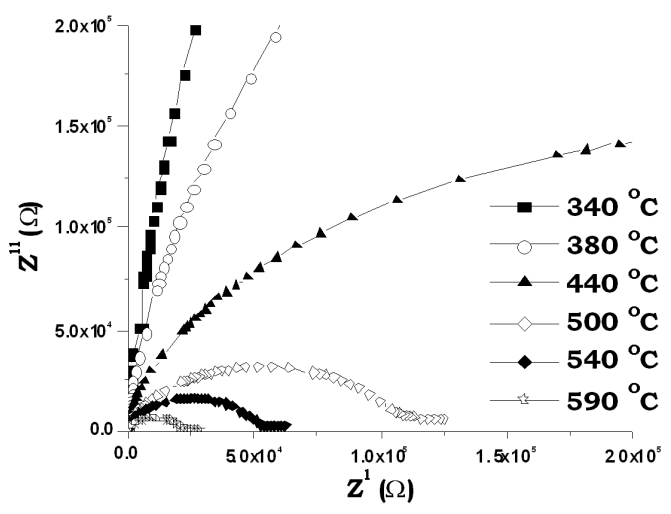

Fig. 9. Complex impedance plane plots at several temperatures.

In Fig. 9, the impedance data of BSBN sample, is presented in the Nyquist diagrams ( $Z^{\prime \prime}$ vs. $\left.Z^{\prime}\right)$ for several representative temperatures. At lower temperatures $<380^{\circ} \mathrm{C}$, the shape of the plots tends to straight line with a large slope indicating the insulator behavior of the sample. At the temperatures at and above $380^{\circ} \mathrm{C}$, the slope of the curve decreases, bowing up to the real axis. At the temperatures $\geq 380^{\circ} \mathrm{C}$ a tendency to a semicircle behavior is observed, indicative of the presence of both localized and non-localized conduction processes.

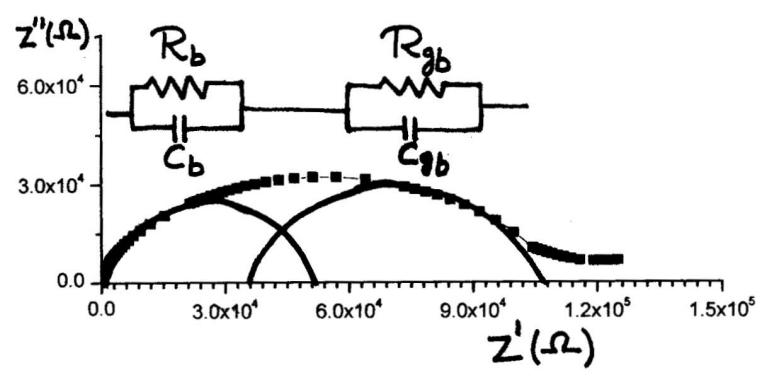

Fig. 10. Cole-Cole plots and equivalent circuit for BSBN showing the electric components of grain and grain boundary.

Figure 10 shows the complex impedance plane plots (the Cole-Cole plots) drawn at $500^{\circ} \mathrm{C}$ for BSBN. The $Z^{\prime}$ vs. $Z^{\prime \prime}$ curve is composed of two semicircles, a small semicircle at high frequencies indicates the effect of grain and large semicircle at low frequencies indicates the grain boundary effect. The intercept of the semicircle on the real axis gives the resistance of grain $\left(R_{\mathrm{b}}\right)$ and grain boundary $\left(R_{\mathrm{gb}}\right)$ of the corresponding component contributing towards the impedance of the sample. The capacitance $(C)$ of each component can be calculated using the formula $\omega \tau=2 \pi f_{\max } R C=1, f_{\max }$ is the frequency of the maximum of the semicircle, $\omega=2 \pi f_{\max }$ and $\tau$ is the relaxation time.

Variation of grain, grain boundary resistance and relaxation time with reciprocal temperature shows the Arrhenius behavior. Grain and grain boundary conduction activation energy $\left(E_{\mathrm{b}}\right.$ and $\left.E_{\mathrm{gb}}\right)$, grain and grain boundary relaxation activation energy $\varepsilon_{\mathrm{g}}$ and $\varepsilon_{\mathrm{gb}}$ have been evaluated and are given in Table I. Activation energies obtained for conduction through grain and grain boundary indicates that the conduction takes place mainly through grain boundaries rather than grains.

Activation energy values $(\mathrm{eV})$ of conduction $(E)$ and relaxation $(\varepsilon)$ for grain $(\mathrm{b})$ and grain boundary $(\mathrm{gb})$.

TABLE I

\begin{tabular}{c|c|c|c|c|c}
\hline \hline Composition & $\begin{array}{c}\text { Temperature } \\
{\left[{ }^{\circ} \mathrm{C}\right]}\end{array}$ & $\begin{array}{c}\text { Grain conduction } \\
\text { activation energy, } \\
E_{\mathrm{b}}[\mathrm{eV}]\end{array}$ & $\begin{array}{c}\text { Grain boundary } \\
\text { conduction activation } \\
\text { energy, } E_{\mathrm{gb}}[\mathrm{eV}]\end{array}$ & $\begin{array}{c}\text { Grain relaxation } \\
\text { activation energy, } \\
\varepsilon_{\mathrm{b}}[\mathrm{eV}]\end{array}$ & $\begin{array}{c}\text { Grain boundary } \\
\text { relaxation activation } \\
\mathrm{energy,}, \varepsilon_{\mathrm{gb}}[\mathrm{eV}]\end{array}$ \\
\hline \multirow{2}{*}{$\mathrm{BSBN}$} & $380-440$ & 0.45 & 0.49 & 0.53 & 0.62 \\
& $480-580$ & 0.51 & 0.60 & 0.43 & 0.51
\end{tabular}




\section{6. $\beta$ parameter}

The electric field relaxation due to ion motion is generally described by the Kohlrausch function $\phi(t)=$ $\exp \left(-\left(t / \tau_{\sigma}\right)^{\beta}\right)(0 \leq \beta \leq 1)[28-31]$, where $\tau_{\sigma}$ and $\beta$ are the conductivity relaxation time and Kohlrausch exponent, respectively. The smaller the value of $\beta$ the greater is the deviation with respect to Debye-type relaxation. The $\beta$ parameter is most often interpreted as a result of correlated motions between the ions. The value of $\beta$ becomes smaller as the cooperation between the charge carriers is more extended. For very small charge carriers concentration, the conductivity is essentially characterized by the independent jumps, whereas when the mobile ion concentration increases, the coupling between charge carriers is more extended. In glasses and crystalline ionic conductors, the value of $\beta$ is close to $0.5[31,32]$.

The value of $\beta$ was determined by fitting the circular $\operatorname{arc} Z^{\prime \prime}=f\left(Z^{\prime}\right)$, the center of semicircle displaced below the $x$-axis. The above fit allows us to determine the value of angle $\phi$ and thus $\beta$ as $\phi=(1-\beta) \pi / 2$. Variation of stretched exponential parameter, $\beta$ with temperature $\left(380-580^{\circ} \mathrm{C}\right)$ in $\mathrm{BSBN}$ which covers $T_{\mathrm{c}}$ of the material is shown in Fig. 11. From Fig. 11 , at $580^{\circ} \mathrm{C}$ the value of $\beta$ is approximately 0.96 , it takes a minimum value about 0.77 at $T_{\mathrm{c}}$, with further increase in temperature the $\beta$ value increases in BSBN. One can conclude from the results that the variation of activation energy and stretched exponential parameter $\beta$ near $T_{\mathrm{c}}$, the dynamic process is different from that of apart from phase transition temperature. According to lattice dynamics theory one of the transverse mode (soft mode) is weakened and the restoring force tends to become zero at ferroelectricparaelectric transition [33]. Hence, if one assumes that the charge carriers couples with soft mode, one may expect that the charge carriers become mobile at $T_{\mathrm{c}}$.

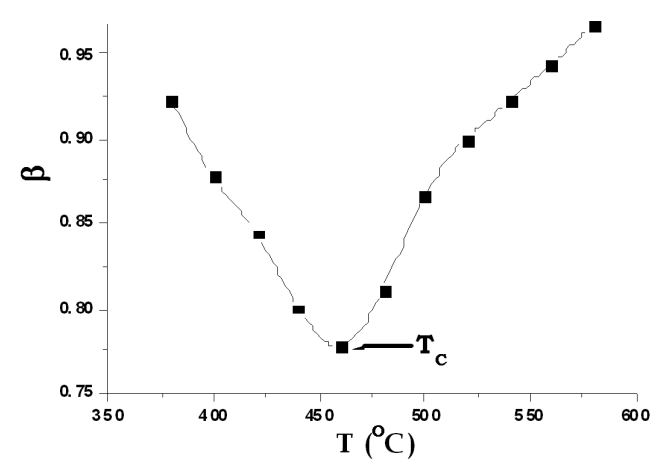

Fig. 11. Variation of stretched exponential parameter, $\beta$ with temperature.

\subsection{Electric modulus}

The electric modulus is the reciprocal of the permittivity $M^{*}=1 / \varepsilon^{*}$. Although it was originally introduced by Macedo et al. [34] to study the space charge relaxation phenomena, $M^{*}$ representation is now widely used to analyze ionic conductivities [35].
Physically, the electric modulus corresponds to the relaxation of the electric field in the material when the electric displacement remains constant, so that the electric modulus represents the real dielectric relaxation process, which can be expressed as [34]:

$$
\begin{aligned}
& M^{*}(\omega)=1 / \varepsilon^{*}(\omega)=M^{\prime}+\mathrm{i} M^{\prime \prime} \\
& \quad=M_{\infty}\left[1-\int_{0}^{\infty}(-\mathrm{d} \Phi / \mathrm{d} t) \exp (-\mathrm{i} \omega t) \mathrm{d} t\right],
\end{aligned}
$$

where $M_{\infty}=\left(\varepsilon_{\infty}\right)^{-1}$ is the asymptotic value of $M^{\prime}(\omega)$, and $\Phi(t)$ is the time evolution of the electric field within the material.

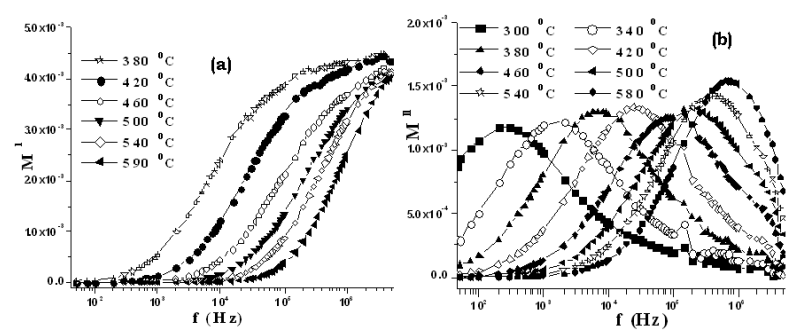

Fig. 12. (a) Real $\left(M^{\prime}\right)$ and (b) imaginary $\left(M^{\prime \prime}\right)$ parts of electric modulus as a function of frequency at different temperatures.

Figure $12 \mathrm{a}$ and $\mathrm{b}$ shows the real and imaginary parts of electric modulus, $M^{\prime}$ and $M^{\prime \prime}$, respectively, as a function of frequency at various temperatures. For each temperature, $M^{\prime}$ reaches a constant value at high frequencies. At low frequencies, $M^{\prime}$ approaches zero, confirming the presence of an appreciable electrode and/or ionic polarization in the studied temperature ranges. $M^{\prime}$ increases from the low frequency of zero toward a high frequency limit $M_{\mathrm{s}}$, and the dispersion shift to the high frequency as increasing temperature.

From Fig. $12 \mathrm{~b}$ at and above $300^{\circ} \mathrm{C}$ peaks appear in $M^{\prime \prime}$, which shifts to higher frequencies with increasing temperature. These peaks indicate the transition from short range to long range mobility with decreasing frequency, where the low frequency side of the peak represents the range of frequencies in which the ions are capable of moving long distances, i.e., performing successful hopping from one site to the neighboring site, whereas for the high frequency side, the ions are spatially confined to their potential wells and can execute only localized motion [36].

We derived the most probable conductivity relaxation times $\tau_{\mathrm{c}}$ from the peak frequency $f_{\max }$ in $M^{\prime \prime}$ based on the condition $2 \pi f_{\max } \tau_{\mathrm{c}}=1$, then plotted their reciprocal temperature dependences. We observe that they follow close to the Arrhenius law $\tau_{\mathrm{c}}=\tau_{0} \exp (E / k T)$. Based on the high temperature data, we obtained the activation energies for the conduction process, in the temperature regions $340-440^{\circ} \mathrm{C}$ and $500-580{ }^{\circ} \mathrm{C}$ they are $0.60 \mathrm{eV}$ and $0.50 \mathrm{eV}$, respectively.

The similar activation energies obtained from $Z^{\prime \prime}$ 
and $M^{\prime \prime}$ plots indicates the localized conduction (dielectric relaxation) of the charge species in the bulk of the sample.

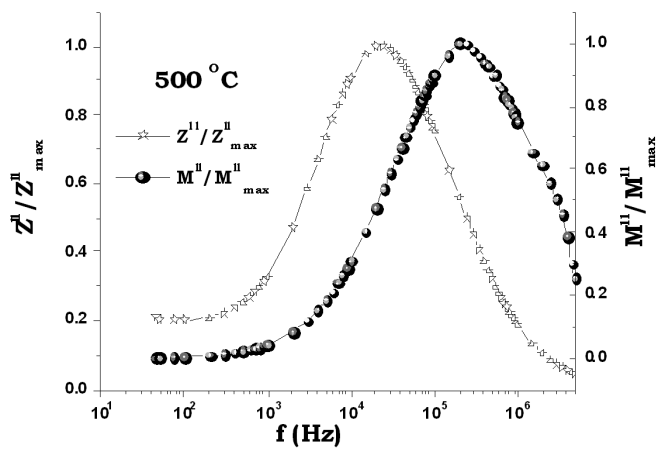

Fig. 13. Normalized imaginary part of impedance $\left(Z^{\prime \prime}\right)$ and normalized imaginary part of modulus $\left(M^{\prime \prime}\right)$ as a function of frequency for BSBN at $500{ }^{\circ} \mathrm{C}$.

Figure 13 shows the variation of $M^{\prime \prime} / M_{\max }^{\prime \prime}$ and $Z^{\prime \prime} / Z_{\max }^{\prime \prime}$ as a function of logarithmic frequency measured at $500^{\circ} \mathrm{C}$. The peak frequency, $f_{M^{\prime \prime}}$, in $M^{\prime \prime} / M_{\max }^{\prime \prime}$ curve is shifted to more high frequency region with relation to one, $f_{Z^{\prime \prime}}$, in $Z^{\prime \prime} / Z_{\max }^{\prime \prime}$ curve. Though these representations it is possible to observe a change of the apparent polarization by the inspection of the magnitude of mismatch between the peaks of both parameters [37].

\section{8. $D C$ and $A C$ conductivity}

Figure 14 shows the variation of real part of ac conductivity with temperature. It can be seen that in the low temperature region there is a distinct dispersion in the values of conductivity. However, as one makes a transition to higher temperatures, the dispersion decreases. This is probably due to the fact that the conductivity is intrinsic at these temperatures. There is a suitable slope change in the conductivity curves around $460^{\circ} \mathrm{C}$, which corresponds to the $T_{\mathrm{c}}$ of the sample. Table II shows the dc and ac conductivity activation energy values of BSBN.

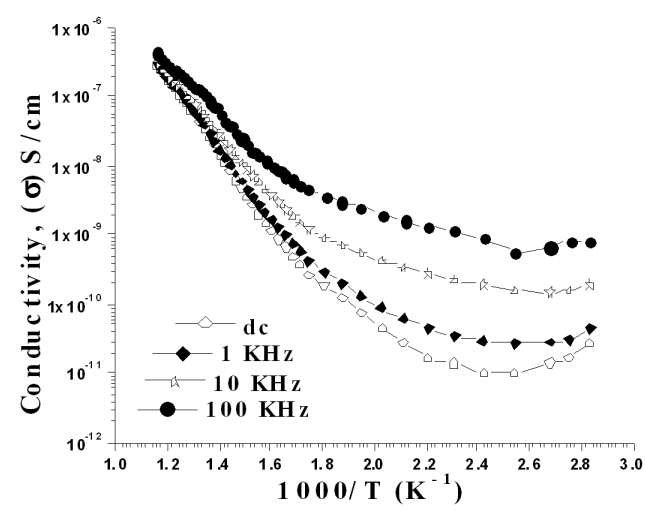

Fig. 14. Arrhenius plots for ac conductivity.
TABLE II

$\mathrm{DC}$ and $\mathrm{AC}$ conductivity activation energy values of BSBN.

\begin{tabular}{c|c|c|c|c}
\hline \hline Composition & \multicolumn{4}{|c}{ BSBN } \\
\hline \multirow{2}{*}{$\begin{array}{c}\text { Temperature } \\
\text { range }\left[{ }^{\circ} \mathrm{C}\right]\end{array}$} & \multicolumn{3}{|c}{ Conductivity activation energy $[\mathrm{eV}]$} \\
\cline { 2 - 5 } & $\mathrm{dc}$ & \multicolumn{4}{|c}{$\mathrm{ac}$} \\
\cline { 3 - 5 } & & $1 \mathrm{khz}$ & $10 \mathrm{khz}$ & $100 \mathrm{khz}$ \\
\hline $580-480$ & 0.50 & 0.50 & 0.37 & 0.39 \\
$440-340$ & 0.60 & 0.61 & 0.55 & 0.58
\end{tabular}

Near $T_{\mathrm{c}}$, in ferroelectric region the activation energies obtained from complex impedance, electric modulus studies and ac conductivity activation energy at $1 \mathrm{kHz}$ are $0.61 \mathrm{eV}, 0.59 \mathrm{eV}$ and $0.61 \mathrm{eV}$, respectively, and are typical values for an ionic conduction in BSBN.

Figure 4 depicts the variation of real part of ac conductivity as a function of frequency at various temperatures. A convenient formalism to investigate the frequency dependence of conductivity in a material is based on the power-law relation proposed by Jonscher,

$$
\sigma_{T}(\omega)=\sigma(0)+A \omega^{n},
$$

where $\sigma_{T}$ is the total conductivity, $\sigma(0)$ is the frequency independent (dc) conductivity, and the coefficient $A$ and exponent $n$ are temperature and material dependent parameters. The term $A \omega^{n}$ comprises the ac dependence and characterizes all dispersion phenomena. The exponent $n$ can vary very differently from material to material, depending on temperature, etc., but it is always such that in general $0 \leq n \leq 1$. For example the value of $n$ is found to be 0.15 at $420^{\circ} \mathrm{C}$ in BSBN. The frequency dependent plateau-like region observed in the low frequency regime can be attributed to the frequency independent conductivity $[\sigma(0)]$.

\section{Conclusions}

The temperature and frequency dependence of the dielectric permittivity, impedance and electric modulus in BSBN have been performed. XRD analysis in BSBN showed the orthorhombic crystal structure. Substitution of $\mathrm{Ba}$ in SBN increases the phase transition temperature of SBN. Variation of critical exponent $n(T)$ with temperature shows a minimum at $T_{\mathrm{c}}$. The Cole-Cole plots of impedance in BSBN ceramic suggest the relaxation to be non-Debye-type. Variation of grain and grain boundary resistance with temperature reflects the fact that the conduction in BSBN is through grain boundary. The value of stretched exponential parameter, $\beta$, showed a minimum at $T_{\mathrm{c}}$ and, on the other hand, it gives higher values, revealing that the transverse mode (soft mode) is weakened and restoring force tends to become zero at ferroelectricparaelectric transition. The shift in $M_{\max }^{\prime \prime}$ indicates the presence of temperature relaxation process in the material. Near $T_{\mathrm{c}}$, in ferroelectric region the activation energies obtained from complex impedance, electric modulus 
studies and ac conductivity activation energy at $1 \mathrm{kHz}$ are $0.61 \mathrm{eV}, 0.60 \mathrm{eV}$ and $0.63 \mathrm{eV}$, respectively, and are typical values for an ionic conduction in BSBN.

\section{References}

[1] B. Aurivillius, Arkh. Khemi 1, 499 (1950).

[2] J.F. Scott, F.M. Ross, C.A. Paz de Araujo, M.C. Scott, M. Huffman, MRS Bulletin 21, 33 (1996).

[3] I.M. Reancy, M. Roulin, H.S. Shulman, N. Setter, Ferroelectrics 165, 295 (1995).

[4] Y. Xu, Ferroelectric Materials and Their Applications North-Holland, Amsterdam 1991.

[5] Y. Noguchi, M. Miyayama, Appl. Phys. Lett. 78, 1903 (2001).

[6] K. Kato, C. Zheng, J.M. Finder, S.K. Dey, T. Torri, J. Am. Ceram. Soc. 81, 1869 (1998).

[7] X. Du, I.W. Chen, J. Am. Ceram. Soc. 41, 3253 (1998).

[8] S.H. Hwang, H.J. Chang, J. Korean Phys. Soc. 42, 139 (2003).

[9] B.H. Park, B.S. Kang, S.B. Bu, T.W. Noh, J. Lee, W. Jo, Nature 401, 682 (1999).

[10] I.W. Kim, C.W. Ahn, J.S. Kim, J.S. Bae, B.C. Choi, J.H. Jeong, J.S. Lee, Appl. Phys. Lett. 80, 4006 (2002).

[11] P.C. Joshi, S.B. Krupanidhi, A. Mansingh, J. Appl. Phys. 72, 5517 (1992).

[12] M. Villegas, A.C. Caballero, C. Moure, P. Durn, J.F. Fernadez, J. Eur. Ceram. Soc. 19, 1183 (1999).

[13] J.F. Scott, Ferroelectric Memories, Springer, Berlin 2000.

[14] D. Dimos, W.L. Warren, H.N. Al-Shareef, in: Thin Film Ferroelectric Materials and Devices, Ed. R. Ramesh, Kluwer, Norwell, MA 1997, p. 199.

[15] E.C. Subba Rao, J. Am. Ceram. Soc. 45, 166 (1962).

[16] G.A. Smolenskii, V.A. Isupov, A.I. Agranovskaya, Fiz. Tverd. Tela 3, 895 (1961).

[17] E.C. Subba Rao, J. Phys. Chem. Solids 23, 665 (1962).
[18] Lu Zhigao, J.P. Bonnet, J. Ravez, P. Hagenmuller, Solid State Ionics 57, 235 (1992).

[19] T.A. Nealon, Ferroelectrics 76, 377 (1987).

[20] A.K. Jonscher, R.M. Hill, C. Pickup, J. Mater. Sci. 20, 4431 (1985).

[21] B. Roling, A. Happe, K. Funke, M.D. Ingram, Phys. Rev. Lett. 78, 2160 (1997).

[22] F.E.G. Henn, J.C. Giuntini, J.V. Zanchetta, W. Granier, A. Taha, Solid State Ionics 42, 29 (1990).

[23] J.C. Giuntini, B, Deroide, P. Belougne, J.V. Zanchetta, Solid State Commun. 62, 739 (1987).

[24] Y. Bensimon, J.C. Giuntini, P. Belougne, B. Deroire, J.V. Zanchetta, Solid State Commun. 68, 189 (1988).

[25] W.K. Lee, J.F. Liu, A.S. Nowick, Phys. Rev. Lett. 67, 1559 (1991).

[26] A.K. Jonscher, Dielectric Relaxation in Solids, Chelsea-Dielectric Press, London 1983.

[27] Z. Lu, J.P. Bonnet, J. Ravez, P. Hagenmuller, Eur. J. Solid State Inorg. Chem 27, 363 (1991).

[28] G. Williams, D.C. Watts, Trans. Fraday Soc. 23, 625 (1970).

[29] K.L. Nagai, S.W. Martin, Phys. Rev. B 40, 10050 (1989).

[30] F.S. Howell, R.A. Bose, P.B. Macedo, C.T. Moynihan, J. Phys. Chem. 78, 639 (1974).

[31] J.M. Reqa, S. Rossignol, B. Tanguy, M.A. Paris, J.M. Rojo, J. Sanz, Solid State Ionics 80, 283 (1995).

[32] N. Zouari, M. Mnif, H. Khemakhem, T. Mhiri, A. Daoud, Solid State Ionics 110, 269 (1998).

[33] Lu. Zhigao, J.P. Bonnet, J. Ravez, J.M. Reau, P. Hagenmuller, Phys. Chem. Solids 53, 1 (1992).

[34] P.B. Macedo, C.T. Moynihan, R. Bose, Phys. Chem. Glasses 13, 171 (1972).

[35] C.A. Angell, Chem. Rev. 90, 523 (1990).

[36] J.S. Kim, J. Phys. Soc. Jpn. 70, 3129 (2001).

[37] R. Gerhardt, J. Phys. Chem. Solids 55, 1491 (1994). 